\title{
Objective Analysis of Pre-Lens Tear Film Stability of Daily Disposable Contact Lenses Using Ring Mire Projection
}

This article was published in the following Dove Press journal: Clinical Optometry

\author{
Sebastian Marx' \\ Julia Eckstein (D) \\ Wolfgang Sickenberger ${ }^{1,2}$ \\ 'JENVIS Research c/o Ernst-Abbe \\ University of Applied Sciences Jena, Jena, \\ Germany; ${ }^{2}$ Department of Optometry \& \\ Vision Science, Ernst-Abbe University of \\ Applied Sciences Jena, Jena, Germany
}

Purpose: The primary objective of this study was to evaluate the in vivo pre-lens noninvasive drying-up time of two types of daily disposable contact lenses (DDCLs) after 12 hours of wear.

Methods: This prospective, randomized, single-center, cross-over pilot study evaluated 31 subjects aged 18-44 years with normal eyes and good tear film stability who were adapted current soft contact lens wearers. Subjects wore nelfilcon A and stenfilcon A DDCLs for 12 hours each on two different days. Non-invasive video keratography drying-up time (NIKDUT) videos of each eye were recorded 12 hours after lens insertion for about 25 seconds, with a 5-minute tear film recovery time allowed between video recordings of the right and left eyes to avoid bias. Post-blink time required to reach $15 \%$ distortion of the projected rings and the speed of break-up at 15 seconds post-blink were measured at each time point and oneye wettability was determined by ring mire projection under white light illumination.

Results: Mean time to reach $15 \%$ ring distortion was similar for nelfilcon A $(19.25 \pm 3.20$ sec) and stenfilcon A (20.24 $\pm 3.02 \mathrm{sec})$ DDCLs but varied highly among subjects. The mean speed of break-up at $15 \mathrm{sec}$ post-blink was $0.3 \pm 0.38 \%$ distortion $/ \mathrm{sec}(95 \%$ confidence interval [CI] $0.138-0.365 \%$ distortion/sec) for nelfilcon $\mathrm{A}$ and $0.2 \pm 0.23 \%$ distortion $/ \mathrm{sec}$ (95\% CI $0.048-0.279 \%$ distortion/sec) for stenfilcon A DDCLs.

Conclusion: Multifunctional topography allowed the objective evaluation of in vivo prelens tear film stability using ring mire projection. This dynamic method was simple, fast and non-invasive, enabling measurements of NIK-DUT and evaluating wettability over a large area, greater than the optical zone of the contact lens surface, for the entire inter-blink interval.

Keywords: pre-lens tear film stability, wettability, daily disposable contact lenses, noninvasive break-up time, non-invasive drying-up time, corneal topography

\section{Introduction}

Both wetting and oxygen permeability are important factors affecting contact lens biocompatibility and comfort. ${ }^{1}$ Wetting by the tear film ensures the integration of the contact lens surface, allowing sliding movement, reducing lid friction on the lens surface, and providing good optical images and regular tear film exchange. ${ }^{2}$ Studies have shown that the main reasons for the termination of contact lens wear are discomfort, dryness, red eyes and vision problems, ${ }^{3-6}$ with wetting of the ocular surface the most important factor preventing discontinuation of contact lens wear. ${ }^{7}$
Correspondence: Sebastian Marx JENVIS Research c/o Ernst-Abbe University of Applied Sciences Jena, CarlZeiss-Promenade 2, Jena 07745, Germany Emails.marx@jenvis-research.com 
Symptoms such as discomfort, dryness, red eyes and vision problems are caused by alterations in tear film composition during contact lens wear. Contact lenses split the tear film into pre-lens and post-lens tear film. Reductions in the lipid layer and aqueous phase of the prelens tear film are dependent on contact lens material and design, ${ }^{8}$ with contact lens wear having a negative effect on tear film stability. ${ }^{8-12}$ Evaporation can result in tear film thinning or break-up, leading to contact lens dehydration, characterized by a water gradient in which there is more moisture on the inner than the outer surface of the lens and more damage to epithelial cells. A thicker lipid layer can provide better tear film stability, resulting in a lower rate of tear film evaporation. ${ }^{9,13,14}$ Tear film break-up time is significantly shorter in eyes wearing contact lenses (3-10 $\mathrm{sec})^{8}$ than in normal eyes without contact lenses $(15 \mathrm{sec}) .{ }^{15}$ Tear meniscus height decreases significantly after 4 hours of working on video display units in both contact lens wearers and non-wearers, with contact lens wearers experiencing enhanced dryness and visual impairment compared with non-wearers. ${ }^{16}$ The increased evaporation on the contact lens surface may also be due to the mechanical effect of the contact lens itself. ${ }^{17}$

Physico-chemical evaluations of the properties of polymer hydrogel lenses have shown that hydrogels act like sponges, resulting in a pressure gradient on the corneal stroma and maintaining a balanced water content. Water content may be reduced by evaporation during the openeye period, the dehydration-rehydration cycle on the front surface of the contact lens caused by blinking and changes in osmotic gradients. ${ }^{18}$ Tear film or the application of rewetting drops may compensate for the lack of moisture in contact lenses. Although this has not yet been shown directly in silicone hydrogel contact lenses, in vitro and in vivo studies have shown that silicone hydrogel lenses dehydrate to a lesser extent than do contact lenses made of other materials. ${ }^{19,20}$

Good wettability requires the surface of the contact lens to be hydrophilic, a condition that results from the accumulation of polar molecular groups on the contact lens surface. Upon insertion into the eye, mucin film forms around the contact lens, with the polymeric surface of the contact lens enhancing wetting. ${ }^{21}$ Under poor wetting conditions, however, hydrogel contact lenses may possess hydrophobic properties, resulting in the alignment of the polar groups to the inside of moist contact lenses.

Wettability is a parameter describing the spread of a drop of liquid over a solid surface. ${ }^{22}$ For complete wetting, the sum of the surface tension of the tear film and the interfacial tension between the lens material and the tear film must be less than or equal to the interfacial tension of the contact lens material. Accordingly, wettability is dependent on the material being wetted and on the composition of the liquid wetting agent. Although contact angle is regarded by contact lens manufacturers as equivalent to wettability, the two parameters are not directly related. $^{18}$

Several techniques, including break-up time, noninvasive break-up time and non-invasive keratography break-up time (NIK-BUT), have been used to assess precorneal tear film stability. ${ }^{23}$ To determine whether a contact lens material is suitable for a subject's tear film conditions, it is necessary to assess wetting at the lens surface. Although this can be determined in vivo using a slit-lamp, this method has the drawback of examining only a small area in the reflex zone, and this area may not be representative of the entire lens surface or optical zone of contact lenses.

In vivo wettability is regarded as an indicator of actual tear film stability while wearing contact lenses. Tear film stability can be determined using invasive and noninvasive methods. Qualitative methods of measuring the surface wetting of contact lenses include determination of pre-lens non-invasive tear film break-up time (PL NITBUT), pre-lens tear film thinning time, interferometry, wave front sensing and high-speed videokeratoscopy. PL NITBUT is based on the method of measuring NITBUT, a marker of tear film stability without lenses. PL NITBUT measures the time from the last complete blink until the first appearance of a distorted grid structure. ${ }^{24}$ It is therefore important to distinguish between actual tear film break-up and tear film thinning of the projected ring structure. ${ }^{25-27}$ Use of the Tearscope and video topography can determine the PL NIBUT non-invasive surface dry-up time. ${ }^{28-34}$ Pre-lens tear film can be analyzed by interferometry, including thin film, Twyman-Green interferometry and lateral sharing interferometry. ${ }^{35,36}$ Furthermore, changes and irregularities in tear film thickness can be evaluated by aberrometry. ${ }^{37}$

The potential of videokeratoscopy is being explored by testing new endpoints, which may provide the contact lens specialist more options to evaluate the surfaces of silicone hydrogel lenses. In the study, which focused on the subjective interpretation of the reflected ring mires, three investigators manually evaluated the first change in wettability on the lens surface after a blink and graded the dewetting appearance in 
five different zones of the lens surface at different time points during the inter-blink period. ${ }^{38}$ In contrast, the present study describes the results of software-supported analyses of the reflected ring mire, a more user independent approach of evaluating the performance of soft contact lens after 12 hours of wear.

Objective determination of tear film break-up time by video topography may enable determination of local drying of the ocular surface. ${ }^{39}$ Technological advances have allowed assessments of a much larger zone than the area around the light reflex, covering more than the optical zone of a contact lens. Multifunctional topographers (Keratograph 5M, Oculus) are simultaneously able to project ring mires onto the lens surface and capture a video of the reflected image (Figure 1). ${ }^{40}$

The primary objective of the study was to compare the tear film stability of nelfilcon A and stenfilcon A daily disposable contact lenses using ring mires projection of the K5M Oculus NIK-BUT Mode after 12 hours of wear. The key variables recorded were time to reach $15 \%$ ring distortion and the speed of tear film break-up.

\section{Materials and Methods}

This study was conducted at a single site in Jena, Germany (JENVIS Research Institute at the Ernst-Abbe University of Applied Sciences Jena), and complied with the ethical principles of the Declaration of Helsinki and Good Clinical Practice (GCP). The study protocol was approved by the local ethic committee of the Friedrich-SchillerUniversität, Jena, Germany, and all subjects provided written informed consent prior to any assessment. The ClinicalTrials.gov identifier is NCT 02089191.

\section{Study Design}

In this prospective, randomized, open-label and subsequently double-masked cross-over pilot study, subjects were randomized to nelfilcon A or stenfilcon A daily disposable silicone hydrogel lenses contact lenses (see Table 1 for lens specifications). Both lens types are CE-marked and commercially available in Europe. Pre-lens tear film stability was assessed as post-blink time required to reach $15 \%$ distortion of the projected rings and the speed of break-up at 15 seconds post-blink measured after $12 \mathrm{hrs}$ of lens wear.

\section{Study Population}

Sample size calculation was based on pilot data obtained for nelfilcon A and stenfilcon A contact lenses for the time from first eye opening post-blink to $10 \%$ and $20 \%$ distortion of the reflected Placido ring system. When the sample size in each group was 28, a 0.050 level one-sided Log rank test for equality of survival curves had $80 \%$ power to detect the difference between the two lenses. To account for possible drop outs, a sample size of 31 subjects was recommended. The study population included volunteer subjects aged 18-44 years who were adapted current wearers of soft contact lenses in both eyes, without dry eye conditions and with good tear film stability $(\geq 13$ seconds). ${ }^{41}$ All subjects had normal eyes, except for requiring correction for refractive error, which can be corrected with spherical lenses to achieve 20/25 VA (inclusion requirement). Subjects with marginal dry eyes were excluded. Subjects were randomly allocated to one of two contact lens materials by a qualified unmasked staff member. Subsequently, both the subject and the investigator
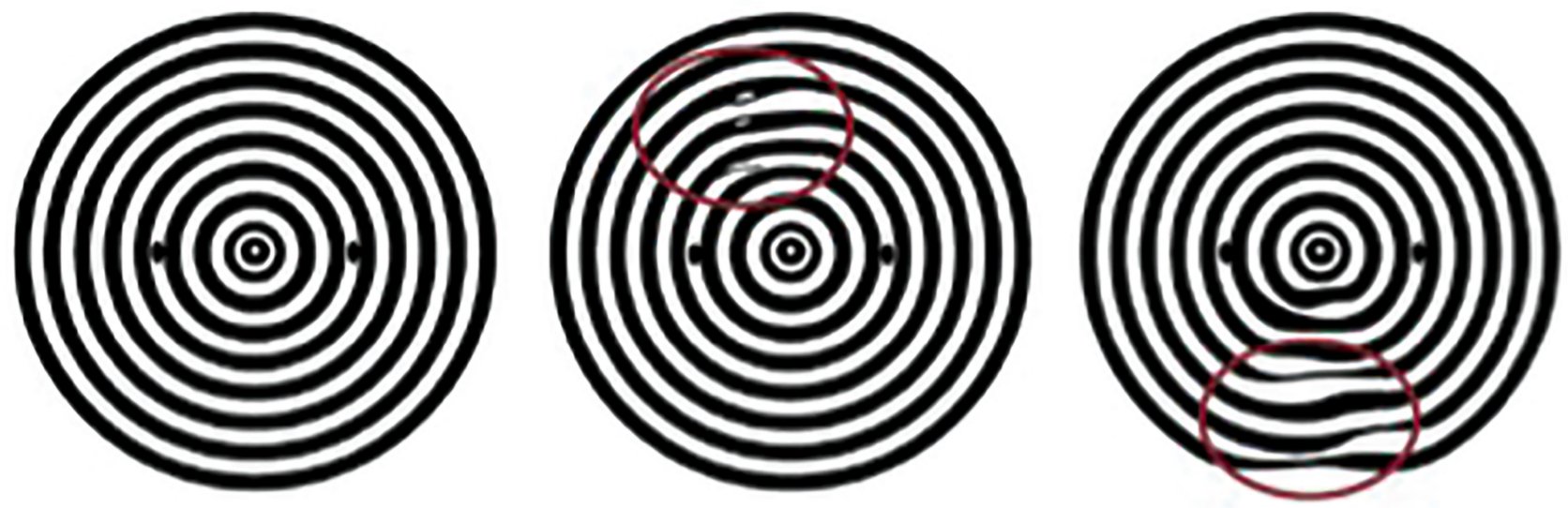

Figure I Graphic of theoretical Placido ring distortions appearing after last blink (left: ideal ring reflection, middle: area with gaps in the projected ring structure, right: wavelike distortions). The red rings highlight areas where distortions (dewetting) has occurred. 
Table I Specifications of Nelfilcon A and Stenfilcon A Daily Disposable Silicone Hydrogel Soft Contact Lenses

\begin{tabular}{|c|c|c|c|c|c|c|}
\hline $\begin{array}{l}\text { Lens Brand } \\
\text { Name }\end{array}$ & $\begin{array}{l}\text { Material } \\
\text { (Manufacturer) }\end{array}$ & $\begin{array}{l}\text { Wettability } \\
\text { Component }\end{array}$ & $\begin{array}{l}\mathrm{H}_{2} \mathrm{O} \text { Content } \\
{[\%]}\end{array}$ & $\begin{array}{l}\text { Rx Power Range } \\
\text { [D] }\end{array}$ & $\begin{array}{l}\text { Base Curve } \\
{[\mathrm{mm}]}\end{array}$ & $\begin{array}{l}\text { Diameter } \\
{[\mathrm{mm}]}\end{array}$ \\
\hline $\begin{array}{l}\text { DAILIES }{ }^{\circledR} \\
\text { AquaComfort } \\
\text { Plus }^{\circledR}\end{array}$ & $\begin{array}{l}\text { nelfilcon A (ALCON } \\
\text { Vision Care Ltd.) }\end{array}$ & $\begin{array}{l}\text { PVA (3I\%), Blister: } \\
\text { PEG, HPMC }\end{array}$ & 69 & $\begin{array}{l}+0.50 \text { to }+6.00 \\
(0.25 \mathrm{D} \text { steps }) \\
-0.50 \text { to }-6.00 \\
(0.25 \mathrm{D} \text { steps) } \\
-6.50 \text { to }-10.00 \\
\text { (0.50 steps) }\end{array}$ & 8.7 & 14.0 \\
\hline MyDay ${ }^{\mathrm{TM}}$ & $\begin{array}{l}\text { stenfilcon A (Cooper } \\
\text { Vision) }\end{array}$ & - & 54 & $\begin{array}{l}+0.50 \text { to }+5.00 \\
(0.25 \mathrm{D} \text { steps }) \\
+5.00 \text { to }+6.00 \\
(0.50 \mathrm{D} \text { steps }) \\
-0.50 \text { to }-6.00 \\
(0.25 \mathrm{D} \text { steps }) \\
-6.50 \text { to }-10.00 \\
(0.50 \text { steps })\end{array}$ & 8.4 & 14.2 \\
\hline
\end{tabular}

were masked to the details of the contact lenses. The duration of the treatment period was 12 hours in a single day, after which the contact lenses were removed. No lens care was required.

Tear film videos were recorded at 12 hours after lens insertion using the multifunctional video topographer (Keratograph $5 \mathrm{M}^{\circledR}$ [K5M] OCULUS Optikgeräte GmbH, Wetzlar, Germany). The test lenses were removed after the $12 \mathrm{~h}$ follow-up visit. Each subject was subsequently crossed over to the other contact lens type on the next day or within the next 6 days, and the procedure was repeated.

Videos of on-eye wettability were captured by ring mire reflex assessment under white light illumination. NIK-DUT videos of each eye were recorded 12 hours after lens insertion, for about 25 seconds each. Subjects were instructed to blink twice and then to suppress blinking for that time. A 5-minute tear film recovery time was allowed between video recordings of the right and left eyes to avoid bias. The videos were analyzed by manufacturer-supplied software to determine ring mire distortion and contact lens surface dry-up time with investigator control. ${ }^{40}$ A grid consisting of eight concentric rings with 12 meridians was overlaid virtually over a video that was recorded during the measurement (Figure 2). This grid divided the area of the front surface of the lens into 192 small segments, which had to be marked subjectively as dewetted in each frame of a video file, ie, 32 times per second. $^{42}$ A trained investigator reviewed each video second by second and checked if the software marked dewetted segments in the analysis area correctly. If a dewetted area was not marked at the correct time point, the investigator edited the video. A data table generated by the grid software allowed determination of the time point at which $15 \%$ ring distortion occurred. A distortion of $15 \%$ of the analyzed area was regarded as clinically relevant in affecting vision (Figure 3 ). ${ }^{43,44}$ No specific data were available to determine another endpoint based on clinical trials rather theoretical consideration.

\section{Statistical Analysis}

The primary analysis population was the per protocol set. The primary outcome variable was time to reach $15 \%$ distortion. Overall analysis was based on the test of

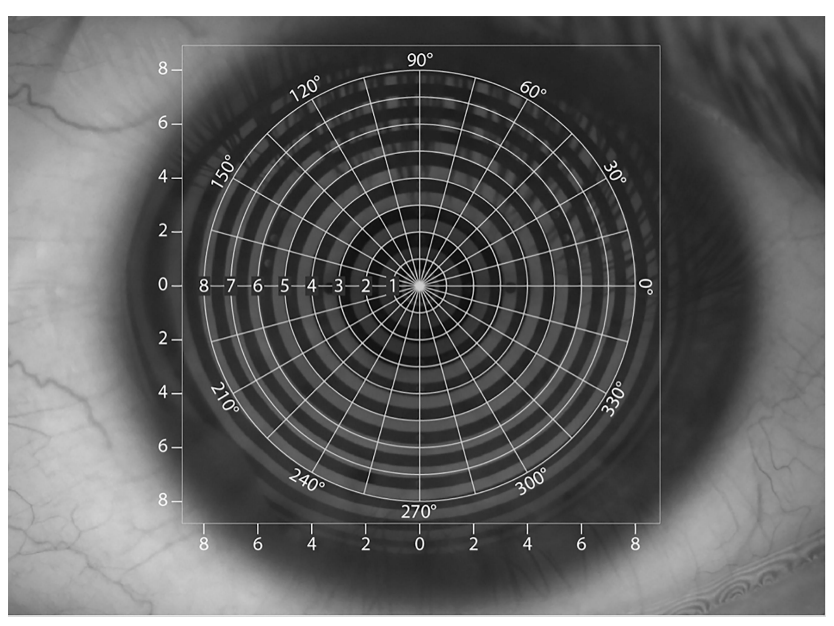

Figure 2 Overlay grid used by the Oculus NIK-BUT software. 


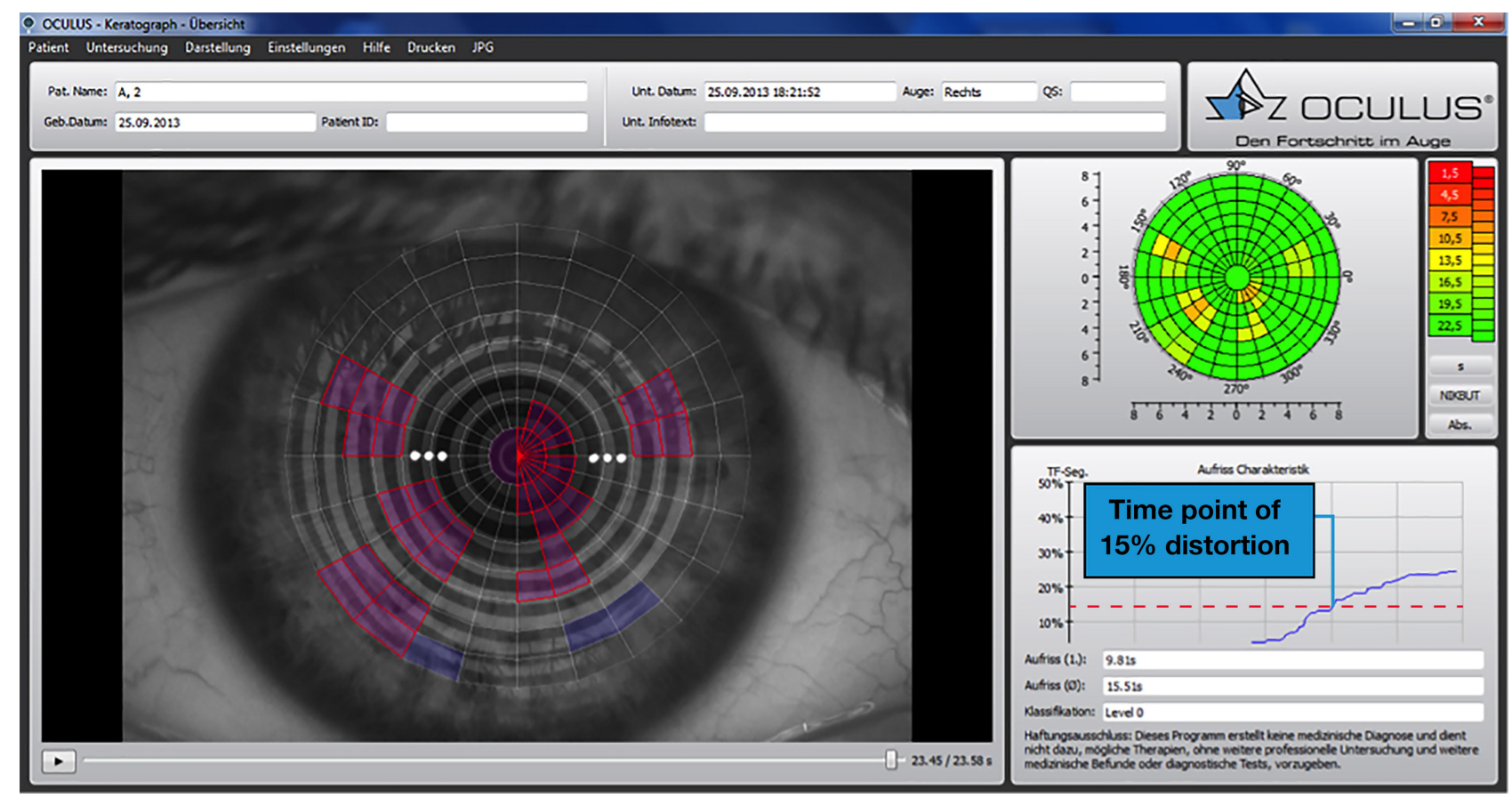

Figure 3 Screenshot of the analyzing software (LEFT: purple segments = objectively detected, blue segments = added by investigator, RIGHT wettability map and function segments over time).

homogeneity with the Log rank test from PROC LIFETEST in SAS. This test was regarded as more appropriate than the Wilcoxon test for these survival data, as the two treatments could differ primarily at longer survival times. Kaplan-Meier plots were also generated.

For the secondary variable, a mixed model repeated measure (MMRM) model was used to analyze the data. The model included terms for period and sequence, as well as lens type (=treatment). An unstructured variancecovariance matrix was used to model the within-subject correlation. If the unstructured variance-covariance matrix resulted in a lack of convergence, then other covariance structures were investigated. Within-treatment and between-treatment estimates of speed of break-up, along with the associated standard errors and 95\% confidence intervals, were calculated.

\section{Results}

Of the 31 subjects with normal eyes and good tear film stability enrolled in this study, 16 (51.6\%) were randomized to nelfilcon A followed by stenfilcon A contact lenses and $15(48.4 \%)$ to stenfilcon A followed by nelfilcon A contact lenses. Across these treatment sequences, 30 $(96.8 \%)$ of the 31 subjects completed the study. One subject was lost to follow up.
Over the 25-second test period, 11 of the 30 subjects wearing nelfilcon $\mathrm{A}$ and 12 of the 30 subjects wearing stenfilcon A lenses for 12 hours reached 15\% distortion of the projected rings, with the majority of subjects not reaching $15 \%$ distortion over the test period. Regardless of lens type, Kaplan-Meier analysis showed good maintenance of tear film coverage during the inter-blink interval, with $60 \%$ and $65 \%$ of subjects wearing nelfilcon A and stenfilcon A contact lenses, respectively, not reaching 15\% segment distortion (Figure 4).

The time to reach $15 \%$ distortion varied widely among subjects, ranging from 12 to 24 seconds post-blink (Table 2). Kaplan-Meier analysis showed that the post-blink time to reach $15 \%$ distortion of the projected rings after 12 hours of lens wear was similar for the two lens types $(p=0.3832$ by $\log$ rank test). Similarly, the mean speed of break-up at 15 seconds post-blink ( $\%$ distortion/sec) after 12 hours of lens wear was similar for subjects wearing nelfilcon A $(0.3 \% / \mathrm{sec})$ and stenfilcon A $(0.2 \% / \mathrm{sec})$ contact lenses (Table 3$)$.

The results of explorative endpoints at the $5 \mathrm{~min}$ and 8 $\mathrm{h}$ follow-up times are not shown. The comfort and vision results were reported previously. ${ }^{38}$ There were no adverse events during the study.

\section{Discussion}

Multifunctional topography units have been shown to successfully measure pre-corneal tear film stability. Use of 


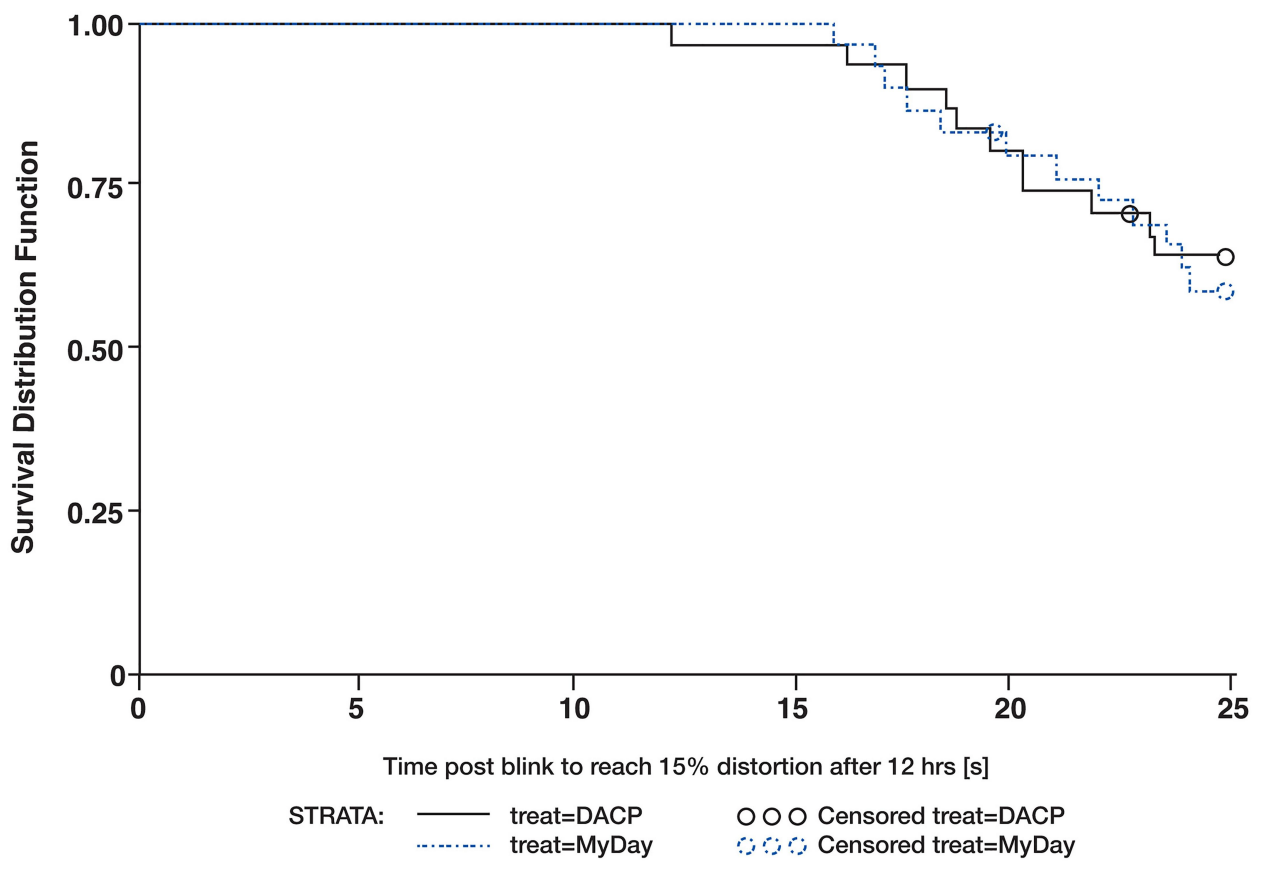

Figure 4 Kaplan-Meier analysis of time post-blink required to reach $15 \%$ distortion after 12 hours of lens wear.

this technology can also determine in vivo pre-lens tear film break-up time using ring mire reflex assessment. This simple, fast and non-invasive technique can be used to evaluate NIK-DUT in an automated, examinerindependent manner. It can be used to assess the wettability of the lens surface over a larger area than the area assessed using slit-lamp-based methods. The area covered by this method is greater than the optical zone of the contact lens surface, measuring an area approximately

Table 2 Time Post-Blink to Reach 15\% Distortion of the Projected Rings After 12 Hours in Subjects Who Reached 15\% Distortion

\begin{tabular}{|l|l|l|}
\hline Seconds & Nelfilcon A & Stenfilcon A \\
\hline N & 11 & 12 \\
Mean (SD) & $19.25(3.20)$ & $20.24(3.02)$ \\
Median & 19.50 & 20.46 \\
Min - Max & $12.2-23.3$ & $15.9-24.1$ \\
\hline
\end{tabular}

Table 3 Mean Break-Up Speed at 15 Sec Post-Blink (\% Distortion/Sec) After 12 Hours of Wear of Nelfilcon $A$ and Stenfilcon A Contact Lenses

\begin{tabular}{|l|l|l|}
\hline \%/Second & Nelfilcon A & Stenfilcon A \\
\hline $\mathrm{N}$ & 31 & 30 \\
Mean (SD) & $0.3(0.38)$ & $0.2(0.23)$ \\
$95 \% \mathrm{Cl}$ & {$[0.138,0.365]$} & {$[0.048,0.279]$} \\
\hline
\end{tabular}

9-10 $\mathrm{mm}$ in diameter and covering approximately 50\% of the contact lens surface. ${ }^{45}$ In theory, this technique can allow the objective quantification of the wettability of different soft contact lens surfaces. The NIK-DUT method does not require contact with the lens. Furthermore, the evaluation can be performed automatically, employing computer software for all measurements and to reduce subjective influences. This provides an advantage over contact angle-based methods, the results of which have low reproducibility. ${ }^{46,47}$

However, this study showed that a totally investigator independent analysis is not yet possible, as almost all videos needed investigator editing. The primary parameter measured in the current study was the post-blink time required to reach $15 \%$ distortion of the projected rings after 12 hours of lens wear. The results obtained with nelfilcon $\mathrm{A}$ and stenfilcon $\mathrm{A}$ lenses did not differ significantly ( $\mathrm{p}=0.3832$ by $\log$ rank test). In addition, the speed of break-up measured 15 seconds after 12 hours of wear did not differ significantly ( $\mathrm{p}=0.1645$ by $\log$ rank test). Both study lenses showed good tear film stability, with most subjects not reaching $15 \%$ distortion of the pre-lens tear film. Moreover, when observed, this level of tear film distortion was reached only about 20 seconds post-blink. Measurements of subjective comfort and vision for nelfilcon A and stenfilcon A contact lenses after 5 minutes, 8 hours and 12 hours of lens wear showed very good 
acceptance of both (data not shown). These findings were confirmed by further studies. ${ }^{48-50}$

Factors that may influence the wettability of daily disposable silicone hydrogel contact lenses include lens composition (ie, the molecular structures of lens materials), environmental and thermal factors (eg, low humidity, air conditioning) and reduced blink rate resulting from increased computer screen-related work. These factors may increase evaporation of the tear film and shorten tear break-up time. ${ }^{51}$

This study had several limitations, generally associated with the use of Placido ring imaging. In general, reflections of Placido ring images on deep-set eyes are not complete, and the quality of these reflected ring images may be disturbed by reflection of the eyelashes, pupil play and mucin structures floating within the tear film directly after the blink, factors that also impede automated analysis. These deficiencies may have led to artifacts falsely indicating a pre-lens tear film break or later detection of a dry spot. The videos should therefore have been reviewed to determine whether the automated software correctly evaluated dewetting of the lens surface. On-eye wettability was determined by ring mire projection under white light illumination using video topography. The white light condition provides higher contrast of the image, but may lead to glare for some patients. In addition, the segments of the overlaid grid were not all equal in size. This may be relevant when the place of the dewetted area is observed. More central segments are smaller in size than more peripheral segments. Practically $15 \%$ of distorted segments would have a greater clinical impact if they occurred more centrally than more inferiorly. Curvature readings would allow a good approximation of the real area expressed in $\mathrm{mm}^{2}$.

Compared with the fully subjective evaluation of the Placido rings reflected from the lens surface, ${ }^{38}$ the objective approach uses a grid that centrally overlies the video. It allows software to highlight detected ring distortion as an indicator of the dewetting process. However, editing by an investigator remains necessary to determine exact results, as real-world limitations like pupil play and initial contact lens movement are challenging factors for fully automated analyses.

\section{Conclusions}

In summary, the wettability parameters of nelfilcon A and stenfilcon A contact lenses did not differ significantly when assessed by the tear film evaluation method of the Oculus K5 keratograph. The technical set-up was able to capture information to assess tear film dynamics over a certain inter-blink period. Although subjective evaluation was able to determine the first noticeable ring distortion and grade wettability, this procedure is very time consuming in practice. ${ }^{38}$ The objective approach may reduce the time necessary to evaluate the captured information. Future studies can evaluate new endpoints that better account for the dynamics of the pre lens tear film and that are not limited to a certain time point as in the present study. The high censoring rate in both lens groups suggests a need for a more sensitive analytic method to better assess the post-blink times of these lenses or an endpoint below 15\% distortion, ideally based on study results indicating a subjective noticeable difference in comfort or vision.

\section{Data Sharing Statement}

The Study results are available on clinicaltrials.gov. https://clinicaltrials.gov/ct2/show/results/NCT02089191.

Due to the varying rights of individuals and contractual rights of parties involved, Alcon does not make a practice of sharing datasets.

\section{Acknowledgments}

The study was funded by Alcon Research, LLC, Fort Worth, TX, USA. None of the authors is employed by Alcon Pharma $\mathrm{GmbH}$ or Novartis AG. Editorial support was provided by BelMed Professional Resources and was funded by Alcon.

\section{Disclosure}

The authors report no conflicts of interest in this work.

\section{References}

1. Guillon M, Maissa C. Use of silicone hydrogel material for daily wear. Cont Lens Anterior Eye. 2007;30(1):5-10. doi:10.1016/j.clae.2006.09. 008

2. Sweeney D. Silicone hydrogels: continuous-wear contact lenses. Butterworth-Heinemann Med. 2004;215.

3. Pritchard N, Fonn D, Brazeau D. Discontinuation of contact lens wear: a survey. Int Contact Lens Clin. 1999;26(6):157-162.

4. Young G, Veys J, Pritchard N, Coleman S. A multi-centre study of lapsed contact lens wearers. Ophthalmic Physiol Opt. 2002;22(6): 516-527.

5. Richdale K, Sinnott LT, Skadahl E, Nichols JJ. Frequency of and factors associated with contact lens dissatisfaction and discontinuation. Cornea. 2007;26(2):168-174.

6. Dumbleton K, Woods CA, Jones LW, Fonn D. The impact of contemporary contact lenses on contact lens discontinuation. Eye Contact Lens. 2013;39(1):93-99.

7. Best N, Drury L, Wolffsohn JS. Predicting success with silicone-hydrogel contact lenses in new wearers. Cont Lens Anterior Eye. 2013;36(5):232-237. 
8. Young G, Efron N. Characteristics of the pre-lens tear film during hydrogel contact lens wear. Ophthalmic Physiol Opt. 1991;11 (1):53-58.

9. Tomlinson A, Cedarstaff TH. Tear evaporation from the human eye: the effects of contact lens wear. J Br Contact Lens Assoc. 1982;5 (4):141-147.

10. Lacrimal Gland. Tear Film, and Dry Eye Syndromes 2. Springer; 1998.

11. Faber E, Golding TR, Lowe R, Brennan NA. Effect of hydrogel lens wear on tear film stability. Optom Vis Sci. 1991;68(5):380-384. doi:10.1097/00006324-199105000-00010

12. Guillon M, Maissa C. Contact lens wear affects tear film evaporation. Eye Contact Lens. 2008;34(6):326-330. doi:10.1097/ICL.0b013e318 $18 \mathrm{c} 5 \mathrm{~d} 00$

13. Nichols JJ, Mitchell GL, King-Smith PE. Thinning rate of the precorneal and prelens tear films. Invest Ophthalmol Vis Sci. 2005;46 (7):2353-2361. doi:10.1167/iovs.05-0094

14. Craig JP, Tomlinson A. Importance of the lipid layer in human tear film stability and evaporation. Optom Vis Sci. 1997;74(1):8-13. doi:10.1097/00006324-199701000-00014

15. Holly FJ, Lemp MA. Tear physiology and dry eyes. Surv Ophthalmol. 1977;22(2):69-87. doi:10.1016/0039-6257(77)90087-X

16. Kojima T, Ibrahim OMA, Wakamatsu T, et al. The impact of contact lens wear and visual display terminal work on ocular surface and tear functions in office workers. Am J Ophthalmol. 2011;152(6):933-940. doi:10.1016/j.ajo.2011.05.025

17. Guillon JP. Tear film structure and contact lenses. In: holly FJ, ed. The preocular tear film in health, disease and contact lens wear. Lubbock TX Dry Eye Institute. 1986;85:914-939.

18. Fatt I. A predictive model for dehydration of a hydrogel contact lens in the eye. $J$ Br Contact Lens Assoc. 1989;12(2):15-31. doi:10.1016/ S0141-7037(89)80017-5

19. Jones L, May C, Nazar L, Simpson T. In vitro evaluation of the dehydration characteristics of silicone hydrogel and conventional hydrogel contact lens materials. Cont Lens Anterior Eye. 2002;25 (3):147-156. doi:10.1016/S1367-0484(02)00033-4

20. Morgan PB, Efron N. In vivo dehydration of silicone hydrogel contact lenses. Eye Contact Lens. 2003;29(3):173-176. doi:10.1097/01. ICL.0000072825.23491.59

21. Benjamin WJ, Piccolo MG, Toubiana HA. Wettability: a blink by blink account. Int Contact Lens Clin. 1984;11(8):492-498.

22. Carre A, Woehl P. Spreading of silicone oils on glass in two geometries. Langmuir. 2006;22(1):134-139. doi:10.1021/la0518997

23. Wiedemann D, Sickenberger W, Michel W, Marx S. Validation of an objective non-invasive method for a qualitative tear film assessment using a corneal topographer. Optom Vis Sci. 2010;87(Suppl):105246.

24. Mengher LS, Bron AJ, Tonge SR, Gilbert DJ. A non-invasive instrument for clinical assessment of the pre-corneal tear film stability. Curr Eye Res. 1985;4(1):1-7. doi:10.3109/02713688508999960

25. Madden RK, Paugh JR, Wang C. Comparative study of two non-invasive tear film stability techniques. Curr Eye Res. 1994;13 (4):263-269. doi:10.3109/02713689408995787

26. Nichols JJ, Sinnott LT. Tear film, contact lens, and patient-related factors associated with contact lens-related dry eye. Invest Ophthalmol Vis Sci. 2006;47(4):1319-1328. doi:10.1167/iovs.05-1392

27. Cheung S-W, Cho P, Chan B, Choy C, Ng V. A comparative study of biweekly disposable contact lenses: silicone hydrogel versus hydrogel. Clin Exp Optom. 2007;90(2):124-131. doi:10.1111/ j.1444-0938.2006.00107.x

28. Bleshoy H, Guillon M, Shah D. Influence of contact lens material surface characteristics on replacement frequency. Int Contact Lens Clinic. 1994;21(5-6):82-95. doi:10.1016/0892-8967(94)90004-3

29. Guillon M, McGrogan L, Guillon JP, Styles E, Maissa C. Effect of material ionicity on the performance of daily disposable contact lenses. Cont Lens Anterior Eye. 2007;90(2):3-8. doi:10.1016/ S1367-0484(97)80029-X
30. Carney FP, Keay L, Stapleton F, Morris CA, Willcox MD. Hydrogel lens wettability and deposition. In Vivo Clin Exp Optom. 1998;81 (2):51-55.

31. Peterson RC, Wolffsohn JS, Nick J, Winterton L, Lally J. Clinical performance of daily disposable soft contact lenses using sustained release technology. Cont Lens Anterior Eye. 2006;29 (3):127-134.

32. Glasson MJ, Stapleton F, Keay L, Willcox MDP.The effect of short term contact lens wear on the tear film and ocular surface characteristics of tolerant and intolerant wearers. Cont Lens Anterior Eye. 2006;29(1):41-47.

33. Wolffsohn JS, Hunt OA, Chowdhury A. Objective clinical performance of 'comfort-enhanced' daily disposable soft contact lenses. Cont Lens Anterior Eye. 2010;33(2):88-92.

34. Subbaraman LN, Glasier MA, Varikooty J, Srinivasan S, Jones L. Protein deposition and clinical symptoms in daily wear of etafilcon lenses. Optom Vis Sci. 2012;89(10):1450-1459.

35. Szczesna-Iskander DH, Iskander DR. Future directions in non-invasive measurements of tear film surface kinetics. Optom Vis Sci. 2012;89(5):749-759.

36. Szczesna DH. Assessing tear film on soft contact lenses with lateral shearing interferometry. Eye Contact Lens. 2011;37(6):342-347.

37. Rae SM, Price HC. The effect of soft contact lens wear and time from blink on wavefront aberration measurement variation. Clin Exp Optom. 2009;92(3):274-282.

38. Muller C, Marx S, Wittekind J, Sickenberger W. Subjective comparison of pre-lens tear film stability of daily disposable contact lenses using ring mire projection. Clin Optom. 2020;12:17-26.

39. Best N, Drury L, Wolffsohn JS. Predicting success with silicone-hydrogel contact lenses in new wearers. Cont Lens Anterior Eye/. 2013;36(5):232-237.

40. Marx S, Sickenberger W. A novel in-vitro method for assessing contact lens surface dewetting: non-invasive keratograph dry-up time (NIK-DUT). Cont Lens Anterior Eye. 2017;40 (6):382-388.

41. Oehring D, Sickenberger W. Prospective Study for Validation and Evaluation of a Newly.developed Procedure for Objective Assessment of Degree of Conjunctival Redness Using a Video Topographer, Science and Technology. Jena (Germany): Ernst-Abbe-University; Master of Science; 2013:321.

42. Kolbe O, Zimmermann F, Marx S, Sickenberger W. Introducing a novel in vivo method to access visual performance during dewetting process of contact lens surface. Cont Lens Anterior Eye. 2020. doi:10.1016/j.clae.2020.02.011.

43. Arnold S, Walter A, Eppig T, Bruenner H, Langenbucher A. Simultaneous examination of tear film break-up and the lipid layer of the human eye: a novel sensor design (Part 1). Z Med Phys. 2010;20(4):309-315.

44. Arnold S, Bruenner H, Langenbucher A. Simultaneous examination of tear film break-up and the lipid layer of the human eye: a novel model eye for time course simulation of physiologic tear film behavior (Part 2). Z Med Phys. 2010;20(4):316-319.

45. Gupta H, Aqil M. Contact lenses in ocular therapeutics. Drug Discov Today. 2012;17(9-10):522-527.

46. Campbell D, Carnell SM, Eden RJ. Applicability of contact angle techniques used in the analysis of contact lenses, part 1: comparative methodologies. Eye Contact Lens. 2013;39(3):254-262.

47. Read ML, Morgan PB, Maldonado-Codina C. Measurement errors related to contact angle analysis of hydrogel and silicone hydrogel contact lenses. J Biomed Mater Res B Appl Biomater. 2009;91 (2):662-668.

48. Fonn D, Dumbleton K. Dryness and discomfort with silicone hydrogel contact lenses. Eye Contact Lens. 2003;29(1 Suppl):S101-S104.

49. Begley CG, Caffery B, Nichols KK, Chalmers R. Responses of contact lens wearers to a dry eye survey. Optom Vis Sci. 2000;77 (1):40-46 
50. Dumbleton K, Woods C, Jones L, Richter D, Fonn D. Comfort and vision with silicone hydrogel lenses: effect of compliance. Optom Vis Sci. 2010;87(6):421-425.
51. Wolkoff P, Nøjgaard JK, Troiano P, Piccoli B. Eye complaints in the office environment: precorneal tear film integrity influenced by eye blinking efficiency. Occup Environ Med. 2005;62(1):4-12.
Clinical Optometry

\section{Publish your work in this journal}

Clinical Optometry is an international, peer-reviewed, open access journal publishing original research, basic science, clinical and epidemiological studies, reviews and evaluations on clinical optometry. All aspects of patient care are addressed within the journal as well as the practice of optometry including economic and business analyses. Basic and clinical research papers are published that cover

Submit your manuscript here: https://www.dovepress.com/clinical-optometry-journal
Dovepress

all aspects of optics, refraction and its application to the theory and practice of optometry. The manuscript management system is completely online and includes a very quick and fair peer-review system, which is all easy to use. Visit http://www.dovepress.com/ testimonials.php to read real quotes from published authors. 